\title{
Erratum: Signature of Energy Losses on the Cosmic Ray Electron Spectrum [Phys. Rev. Lett. 125, 051101 (2020)]
}

\author{
Carmelo Evoliø, Pasquale Blasi, Elena Amato, and Roberto Aloisio
}

(Received 20 May 2021; published 15 June 2021)

DOI: 10.1103/PhysRevLett.126.249901

Thanks to the precise measurements of AMS-02 a new feature in the cosmic-ray electron (CRE) spectrum was identified in [1]. The new feature cannot be attributed to the contribution of a new component (producing electrons and positrons in equal amount), as PWNe, since this contribution is severely constrained by the measurements of the positron spectrum [2].

In our Letter we concluded that the feature in the CRE spectrum is due to the transition from Thompson to Klein-Nishina $(\mathrm{KN})$ of the inverse Compton energy losses of electrons on the UV background. This result was obtained by adopting a handy parametrization of the KN transition first put forward in Ref. [3].

Following our Letter, the authors of [4] noticed that the feature in the electron spectrum that we reported in [2] is magnified by having adopted a poor approximation of the inverse compton (IC) loss rate [3] [see Eq. (5) in the main paper [2]]. That, the authors conclude, casts doubt on our interpretation of the feature measured by AMS-02 in the electron spectrum in terms of transition from the Thomson to $\mathrm{KN}$ regime. The authors also propose an improved parametrization for the IC cross section [Eq. (5) of [4] ]. We checked that such a revised parametrization indeed provides a better description of the transition to the $\mathrm{KN}$ regime.

We have addressed the criticism of [4] in detail in a recent article [5] where we adopted the KN transition as in Eq. (5) of [4]. While we confirm that with the same simplistic assumptions adopted in Ref. [2] the feature gets considerably smoothed, we also find that the feature becomes prominent when (i) the UV background is properly estimated as a volume average over the propagation region of electrons of given energy, and (ii) the spiral distribution of the sources in the Galaxy is taken into account. None of these two conditions would be sufficient, by itself, to reproduce the feature in the CRE spectrum.

In Fig. 1 we show the updated plots which replace Fig. 1 and Fig. 2 of the main text of [2], as already presented in [5], where a detailed discussion of all relevant physics was presented. In the left panel we show the timescale for electron energy losses, where the UV was chosen so as to be the volume average over the region that the electrons diffuse on. One can appreciate that the feature remains quite evident in the energy loss timescale (which is insensitive to the spatial distribution
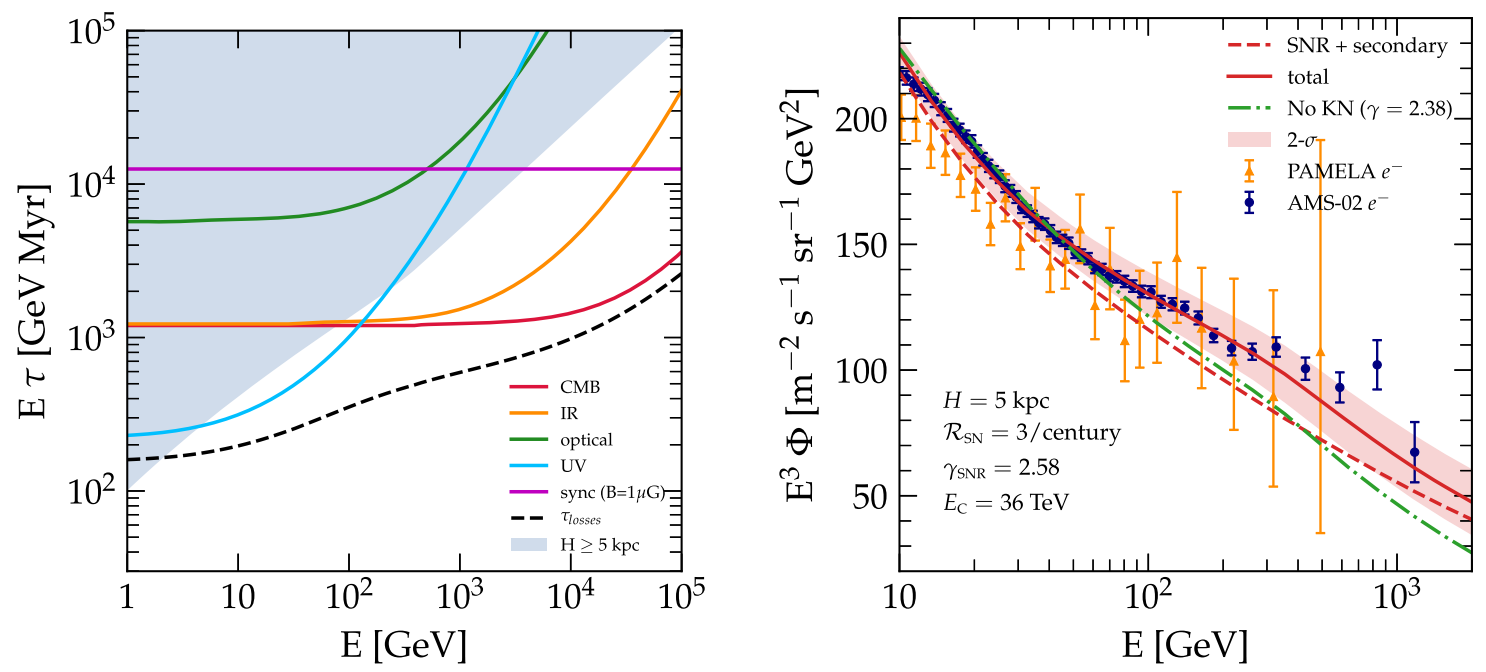

FIG. 1. Left: energy loss timescale for CREs as a function of energy during their propagation in the Galaxy. The timescales are multiplied by $E$ to give prominence to the deviations from the standard $b \propto E^{2}$ regime (reproduced from [5]). Right: electron flux from SNRs is shown with a red dashed line, and the total flux of electrons (including pulsars and secondary products of CR interactions) with a red solid line. The total flux obtained neglecting the KN effect (dash-dotted green line) is also shown (reproduced from [5]). 
of sources). In the right panel, we show that the feature in the total CRE spectrum is still present (red solid line). The crucial role played by the transition to $\mathrm{KN}$ is clear if one compares the solid red line with green dash-dotted line, which shows the same total CRE spectrum but ignoring, in an artificial way, the transition to $\mathrm{KN}$.

We conclude that although the technical point raised by [4] is correct and the parametrization of the IC scattering cross section as proposed by [3] is not accurate enough to describe the transition to the $\mathrm{KN}$ regime, the appearance of a feature of $\gtrsim 40 \mathrm{GeV}$ in the CRE spectrum due to such transition remains solid, as shown in Fig. 1 and discussed in detail in [5].

[1] M. Aguilar and AMS Collaboration, Phys. Rev. Lett. 122, 101101 (2019).

[2] M. Aguilar and AMS Collaboration, Phys. Rev. Lett. 122, 041102 (2019).

[3] R. Schlickeiser and J. Ruppel, New J. Phys. 12, 033044 (2010).

[4] K. Fang, X.-J. Bi, S.-J. Lin, and Q. Yuan, Chin. Phys. Lett. 38, 039801 (2021).

[5] C. Evoli, E. Amato, P. Blasi, and R. Aloisio, Phys. Rev. D 103, 083010 (2021). 